\title{
The Design and Implementation of Color-Matching CAD Software Based on Elementary-Weave-Structured Woven Fabric
}

\author{
Xiongjun Zhu *1, a, Zhenhua Bao ${ }^{2, b}$ \\ ${ }^{1}$ Department of Computer Science, Wuhan Polytechnic, Wuhan 430074 ,China \\ ${ }^{2}$ School of Light Industry, Textile\&Clothing Engineering, Wuhan Polytechnic, Wuhan 430074,China \\ azhuxiongjun@126.com, 'zhb2004129@163.com
}

Keywords: elementary weave structure, woven fabric, color-matching CAD, software development.

\begin{abstract}
In response to the need of woven fabric color-matching CAD software in small and medium enterprises, the article proposes the implementation of software system based on elementary weave structure. Using Microsoft visual $\mathrm{C}++6.0$ application development platform, through the blending ratio of the fabric, color mixing effect calculation to develop a system which can rapidly match colors to the warp and weft fabrics and organizational structure changes. The results indicate that the software is easy to program and small pieces of weave fabric samples have tested its viability. The software can fast, conveniently and accurately simulate the effect of color matching.
\end{abstract}

\section{Introduction}

In the textile and garment industry, computer-aided product design has become increasingly important during the process of production. At present, the textile CAD / CAM technology is widely used in product design. However during the production, designers cannot know in advance the partial or overall color effect of the product based on the colors of fabric warp and weft yarns. They can only rely on their experience as designers, thus they have to constantly change the color of yarns so as to achieve the anticipated overall effect of the product.

With small increase in cost, how to quickly and easily choose the color of yarn and ensure that the color-matching project can meet the demand of customers and market is the focus of small companies now.

Woven fabric color-matching software is necessary for all the small and medium enterprises who don't have sufficient conditions and who have to predict a rather certain color-matching effect before sampling. The establishment of the fabric color-matching system which adapts rapid color changes of weft and yarn in the organizational structure is beneficial.

\section{Theoretical basis[1,2]}

Fabrics. The product made of yarns which are arranged according to certain rules as two mutually perpendicular groups in weaving loom is known as woven fabrics, or referred to as fabric. On the fabric, the thread which is parallel to the longitudinal (or parallel to the direction of weaving machine) is called the warp yarn (line), and the thread which is vertical to the margin of the fabric (or vertical to the direction of loom machine) is known as the weft (line). Warp and weft will be interwoven to form a fabric.

The concept of fabric weaves and weave cycle. The rule according which warp and weft cross each other regularly in the fabric is called the fabric weave. When warp moves underneath the weft, or the other way around, warp and weft will cross once. When weft moves underneath warp or the other way around, they will be united thus forming the fabric. The intersection where warp and weft cross each other is a weave point (floating point). 
When the floating point is on the weft, it is called warp weave point. If it is on the warp, it will be called weft weave point. When the two points start to appear regularly according to a certain rule, it is called a weave cycle.

In this cycle, the number of vertical grid represents the number of weave cycle of warp (Rj), the order is from left to right; the number of horizontal grid represents the number of weave cycle of weft $(\mathrm{Rw})$, and it is from top to bottom.

Woven fabric structure. Fabrics which meet all the following conditions are woven fabrics: Number of weave point is a constant, namely, $\mathrm{S}=$ constant;

On each warp or weft, there is only one warp (weft) weave point, others are weft (warp) weave points. The law of the movement of warp and weft is usually demonstrated by chart in which the vertical grids indicate the warp while the horizontal grids indicate the weft.

Woven fabric including three weave: plain, twill and satin is the basis all kinds of fabrics. Shown in Figure 1.

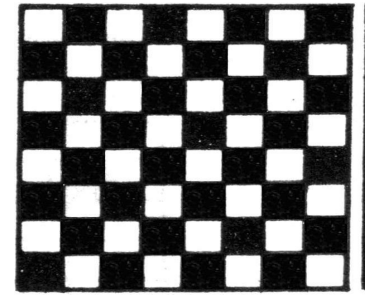

Plain weave

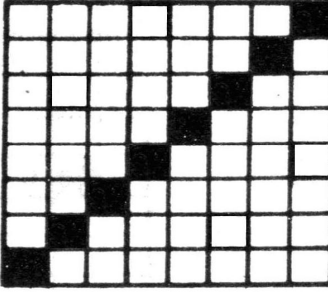

Twill weave

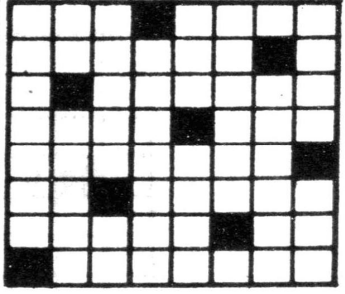

Satin weave

Fig. 1: Three basic woven fabrics

\section{Color-matching CAD software algorithm design}

Calculation of the fabric warp and weft. Pattern and color of a textile product is shown by the intertwined warp and weft as well as the different colors of warp and weft used in fabric. Different fabric structure, and different arrangement of the yarns will result in great difference in weave fabric patterns and color effects of the product[3].

Due to the different structure of fabrics, woven warp and weft will display different color effects in different structures. The surface of a product of warp structure can reflect a color sense dominated by the color of warp yarns, and it is the same for a weft structure product. Therefore, the approximate square or near square color fabric, the mix ratio of warp and weft can be calculated according to the following formula [4]:

$$
\begin{gathered}
c_{j}=\frac{t_{j}}{r_{j} \times r_{w}} \\
c_{w}=\frac{t_{w}}{r_{j} \times r_{w}}
\end{gathered}
$$

In the formula: cj, cw_- The percentage of the warp or weft in the entire weave;
$\mathrm{tj}$, tw- The warp, weft weave points;
rj, rw-The number of warp, weft cycle weave

Calculation of the fabric color mixing effect. According to the space theory of light, the following calculation can simulate the effect of fabric color mixing effect[5]:

$$
\begin{aligned}
& (R, G, B)=c_{j} \times\left(R_{j}, G_{j}, B_{j}\right)+c_{w} \times\left(R_{w}, G_{w}, B_{w}\right) \\
& R=c_{j} \times R_{j}+c_{w} \times R_{w} \\
& G=c_{j} \times G_{j}+c_{w} \times G_{w} \\
& B=c_{j} \times B_{j}+c_{w} \times B_{w}
\end{aligned}
$$


In the formula $\mathrm{R}, \mathrm{G}, \mathrm{B}$ — color value of mixed colored warp,weft Textile

$\mathrm{R}_{\mathrm{j}}, \mathrm{G}_{\mathrm{j}}, \mathrm{B}_{\mathrm{j}}$-Warp color value;

$\mathrm{R}_{\mathrm{w}}, \mathrm{G}_{\mathrm{w}}, \mathrm{B}_{\mathrm{w}}-$ Weft color value。

\section{Design and implementation of color-matching CAD software program}

Using Microsoft visual $\mathrm{C}++6.0$ programming language, based on color matching software algorithm to complete the software design. Software program can work in the situation when changed color values of the warp and weft and different fabric structures lead to the changing color-matching effects. Changing the fabric structure will result in a changing space mixing effect, namely, the appearance of the colored fabric.

The Color-matching CAD software program design process is shown as following:

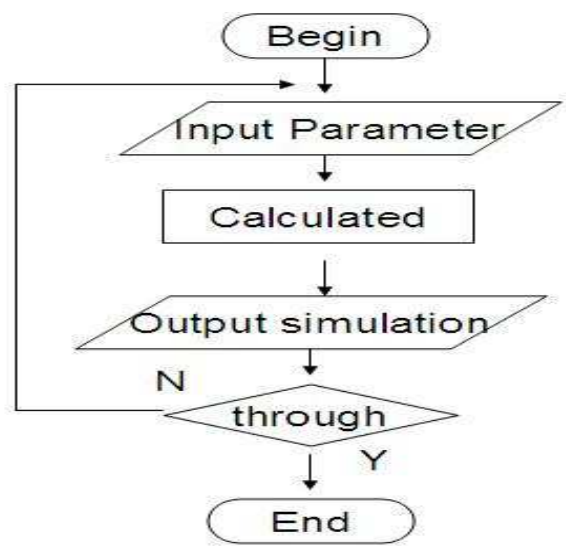

Fig. 2: Color-matching CAD program design process

The realization of color-mix ratio of fabric. Start $\mathrm{VC}++$, use AppWizard create a new MFC AppWizard project,choose "Dialog Based",then add the resources needed to program[6,7]. The following are the relevant part code.

void CColorDlg::DoDataExchange(CDataExchange* pDX)

\{

CDialog::DoDataExchange(pDX);

$/ /\{\{$ AFX_DATA_MAP(CColorDlg)

DDX_Text(pDX, IDC_t $\left.\mathrm{j}, \mathrm{m}_{-} \mathrm{tj}\right)$;

DDV_MinMaxInt $\left(\mathrm{pD} \overline{\mathrm{X}}, \mathrm{m}_{-} \mathrm{t} \mathrm{j}, 1,10\right)$;

DDX_Text(pDX, IDC_tw, m_tw);

DDV_MinMaxInt(pDX, m_tw, 1, 10);

DDX Text(pDX, IDC Rw1, m_Rw1);

DDV_MinMaxInt(pDX, m_Rw1 $, 0,255)$;

DDX_Text(pDX, IDC_Gj, m_Gj);

DDV_MinMaxInt(pDX, m_Gj, 0, 255);

DDX_Text(pDX, IDC_Gw, m_Gw);

DDV_MinMaxInt(pDX, m_Gw, 0, 255);

DDX_Text(pDX, IDC_Bj, m_Bj);

DDV MinMaxInt(pDX, m Bj, 0, 255);

DDX_Text(pDX, IDC_Bw, m_Bw);

DDV_MinMaxInt(pDX, m_Bw, 0, 255); 
Fabric color-mixing effect. According to formula (1)(2), we have calculated the value of the $c_{j}, c_{w}$. According to formula (3)(4)(5), we have calculated the final RGB value.

Before the drawing, using MFC base class library of the Cbrush, displaying mixed color effect. The following is the relevant code.

void CColorDlg::OnPaint( )

\{

if (IsIconic( ))

\{

CPaintDC dc(this); // device context for painting

SendMessage(WM_ICONERASEBKGND, (WPARAM) dc.GetSafeHdc( ), 0);

// Center icon in client rectangle

int cxIcon $=$ GetSystemMetrics(SM_CXICON);

int cyIcon $=$ GetSystemMetrics(SM_CYICON);

CRect rect;

GetClientRect(\&rect);

int $\mathrm{x}=($ rect.Width ()$-\operatorname{cxIcon}+1) / 2$;

int $y=($ rect.Height ()$-$ cyIcon +1$) / 2$;

// Draw the icon

dc.DrawIcon(x, y, m_hIcon);

\}

else

\{

CDialog::OnPaint( );

\}

void CColorDlg::OnButtonShow( )

\{

// TODO: Add your control notification handler code here

CWnd $*$ pWnd $=$ GetDlgItem(IDC_Color); //IDC_STATIC1 specified in the dialog editor $\mathrm{CDC}^{*} \mathrm{pControlDC}=\mathrm{pWnd}->\operatorname{GetDC}(\overline{)}$;

pWnd->Invalidate( );

pWnd->UpdateWindow( );

UpdateData( );

int $\mathrm{R}, \mathrm{G}, \mathrm{B}$;

$\mathrm{R}=\mathrm{m} \_\mathrm{tj} /\left(\mathrm{m}_{-} \mathrm{tj}+\mathrm{m} \_\mathrm{tw}\right) * \mathrm{~m} \_\mathrm{Rj} 1+\mathrm{m}_{-} \mathrm{tw} /\left(\mathrm{m}_{-} \mathrm{tj}+\mathrm{m} \_\mathrm{tw}\right) * \mathrm{~m} \_\mathrm{Rw} 1$;

$\mathrm{G}=\mathrm{m}_{-} \mathrm{tj} /\left(\mathrm{m}_{-} \mathrm{tj}+\mathrm{m}_{-} \mathrm{tw}\right) * \mathrm{~m}_{-} \mathrm{Gj}+\mathrm{m}_{-} \mathrm{tw} /\left(\mathrm{m}_{-} \mathrm{tj}+\mathrm{m}_{-} \mathrm{tw}\right) * \mathrm{~m}_{-} \mathrm{Gw}$;

$B=m_{-} t j /\left(m_{-} t j+m_{-} t w\right) * m_{-} B j+m_{-} t w /\left(m_{-} t j+m_{-} t w\right) * m_{-} B w ;$

CBrush brush;

brush.CreateSolidBrush(RGB(m_Rj1,m_Gj,m_Bj));

pControlDC->SelectObject(\&brush);

pControlDC->Rectangle(20,20,80,60);

brush.CreateSolidBrush(RGB(m_Rw1,m_Gw,m_Bw));

pControlDC->SelectObject(\&brush); 
pControlDC->Rectangle(130,20,190,60); brush.CreateSolidBrush(RGB(R,G,B));

pControlDC->SelectObject(\&brush);

pControlDC->Rectangle $(240,20,300,60)$;

pWnd->ReleaseDC(pControlDC); \}

\section{Color-matching CAD software Interface:}

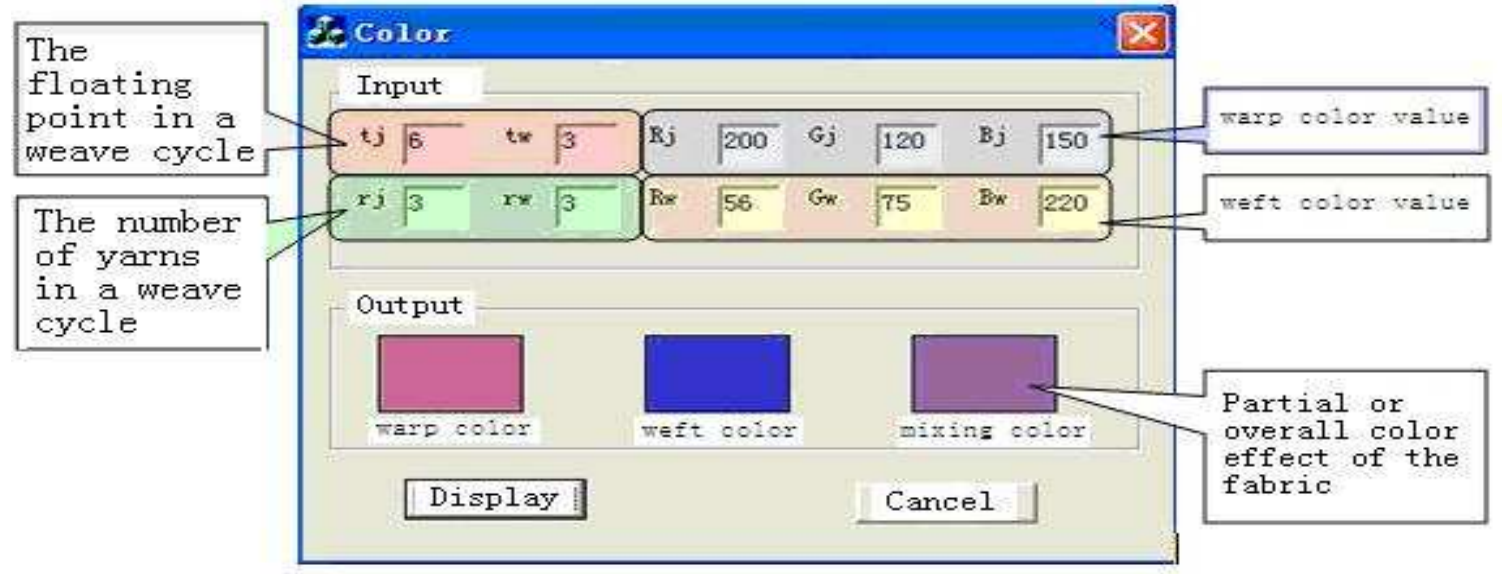

Fig. 3 Color-matching CAD software Interface

\section{Conclusion}

Using fabric sample, picture on the left shows the simulation of the result of color-matching system, picture on the right shows the real fabric sample.

For plain weave, $t_{j}=t_{w}=2, r_{j}=r_{w}=2$, identifying the warp color value $(851,13,510)$, weft color value $(248,420,515)$.

Inputting the parameters into the color-matching system, we will get the color-mixing effect of the fabric. Fabric sample and simulation results validate the practical effects of the software.

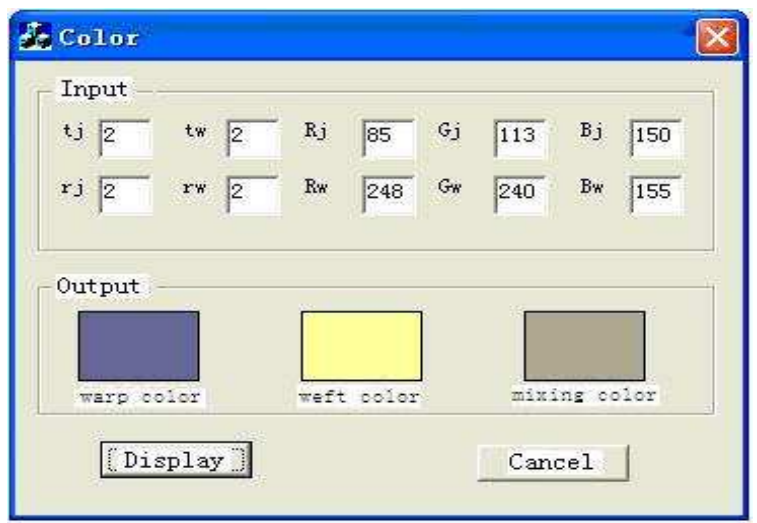

Fig. 4 Stimulated color-matching effect

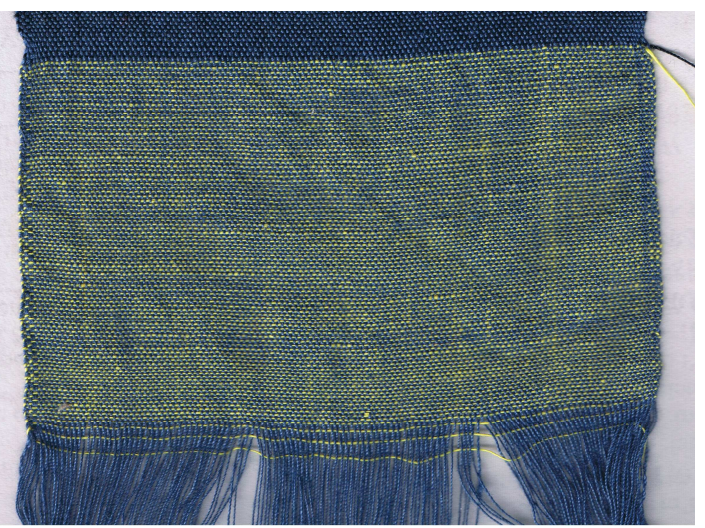

Fig. 5 Sample fabric

Below is another comparison group. It can be seen from the figure that the software simulation results and the physical effect of the sample are almost consistent. 


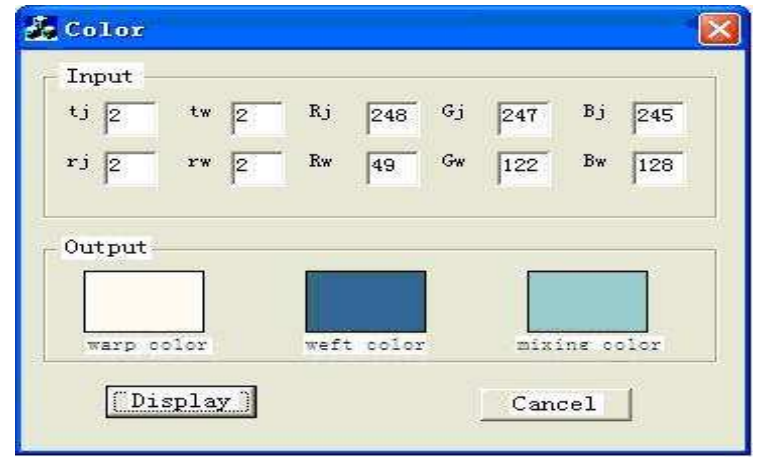

Fig. 6 Stimulated color-matching effect

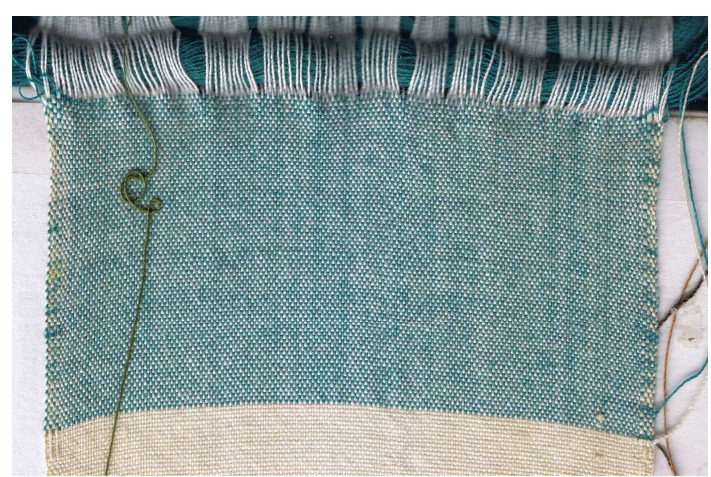

Fig. 7 Sample fabric

\section{Acknowledgment}

This thesis is one in the series of research in the project of "the application of computer color-matching in the textile pattern design" initiated by the Education Department of Hubei Province, issue number: Q200750001/2007SK173. This software has been applied into actual production of school mill, and has proved its practical value.

Xiongjun Zhu(1966-),male,Wuhan polytechnic associate professor,Email:zhuxiongjun@126.com

\section{References}

[1] Cai Bixia, in: Fabric structure and design, edited by China Textile \& Apparel Press, Beijing(2004),in press.

[2] Bao Zhenhua in:Jacquard Technoloqy and Fabric CAD, edited by China Textile \& Apparel Press,Beijing(2009),in press.

[3] Bao Zhenhua, Xu Hua and Wang Zuohong: Research on Applications of Computer Color Matching Technology in Textiles Pattern Design. Journal of South - Central University for Nationalities (Nat.Sci.Edition).V01.29(2010), p.107

[4] Huang CuiRong, in: Textile material design, edited by China Textile \& Apparel Press, Beijing(2007), in press.

[5] Ruan QiuQi, in: Digital image processing learn, edited by Publishing House of Electronic Industry, Beijing(2001), in press.

[6] Lang Rui, in: Digital image processing learn-Visual $C++$ realized, edited by Beijing Hope Electronic Press, Beijing(2001), in press.

[7] Tan JinMing, in: Visual C++ Graphics programming skills and examples, edited by Posts \& Telecom Press, Beijing(2002), in press. 